\title{
Multinucleated cells are involved in normal development and apoptosis in mouse testes
}

\author{
LAN LUO $^{1 *}$, YUHONG LI $^{2 *}$, YULING YANG $^{1}$, YONGSHU HE $^{1}$, YIN WANG $^{2}$, ZHONGYI XU $^{1}$ and YAN ZHANG ${ }^{1}$ \\ ${ }^{1}$ Department of Cell Biology and Medical Genetics, Kunming Medical University, Kunming, Yunnan 650500; \\ ${ }^{2}$ Laboratory of Cranial Cerebral Disease, Department of Neurobiology of Basic Medical College, \\ Center of Scientific Technology, Ningxia Medical University, Yinchuan, Ningxia 750004, P.R. China
}

Received March 1, 2013; Accepted June 19, 2013

DOI: $10.3892 / \mathrm{mmr} .2013 .1568$

\begin{abstract}
Multinucleated cells are present in impaired spermatogenesis and in the senescent testis. Following accumulating evidence from our previous studies on the identification of multinucleated cells during normal testicle development, the current study further investigated the possible mechanism and role of these multinucleated cells. Healthy male Kunming mice were used in the present study. The association between multinucleated cells and cell apoptosis were analyzed using TUNEL analysis and immunohistochemistry. The results showed that multinucleated cells are widespread in the testicular tissue of seminiferous tubules on postnatal days 23 , $27,30,33,36,40,47,50$ and 54 suggesting that these cells are involved in the process of normal development of mouse testis. Histochemical analysis revealed a lack of proliferating cell nuclear antigen, cyclin D1 protein expression in multinucleated cells, suggesting that these cells are not involved in the G1 and $\mathrm{S}$ phases of the cell cycle and cell proliferation. Increased expression of Bax and caspase 3 was detected, revealing that multinucleated cells may be associated with cell apoptosis during testicular development. To the best of our knowledge, this study demonstrated for the first time that multinucleated cells are present during normal testicular development and may be associated with spermatogonial stem cell apoptosis. Therefore, multinucleated cells may be important in the spermatogenesis process.
\end{abstract}

Correspondence to: Professor Yan Zhang, Department of Cell Biology and Medical Genetics, Kunming Medical University, 1168 West Chunrong Road, Kunming, Yunnan, P.R. China E-mail:976153438@qq.com

Professor Yin Wang, Department of Neurobiology, Ningxia Medical University, 1160 Shengli Street, Yinchuan 750004, P.R. China

E-mail: yin-wang@hotmail.com

*Contributed equally

Key words: apoptosis, multinucleated cells, testicular development

\section{Introduction}

Spermatogenesis is composed of three phases, mitosis, meiosis and spermiogenesis, through which spermatogonial stem cells (SSCs) self-renew and differentiate into sperm. To maintain normal homeostasis, the balance between self-renewal and differentiation of SSCs must be closely regulated. Increasing evidence has revealed that the processes of maintenance and self-renewal of SSCs and testicular development are regulated by a variety of factors and involves the participation of multiple genes $(1,2)$.

Multinucleated cells are present in a variety of sites and in association with several conditions. Coenocytes have been identified in specific pathological processes, including cells affected by chemicals, drug injury or exposure to adverse factors, including thermal or mechanical damage (3-9). However, the involvement of multinucleated cells in normal testicular development requires further clarification. However, the appearance of multinucleated cells in normal testicular development processes has not been reported.

In the present study, the possible mechanism and role of multinucleated cells in normal testicular development was investigated. Results revealed that multinucleated cells occur during the mouse testicular development process. To explore the mechanism of the occurrence of multinucleated cells in testicular development, the expression of cell proliferation- and apoptosis-related proteins, including proliferating cell nuclear antigen (PCNA), cyclin D1, caspase 3 and Bax was detected in multinucleated cells of mouse testicle tissue (day 30 and 33 following birth).

\section{Materials and methods}

Animals. Experiments were conducted following the Guide for Care and Use of Laboratory Animals (NIH Guide). Protocols for the use of animals were approved by the Department of Laboratory Animal Sciences (Kunming Medical University, Kunming, China). Experimental healthy male Kunming mice were purchased from the Laboratory Animal Center of Kunming Medical University and were divided into the following groups: postnatal day $1,4,8,11$, $15,20,23,27,30,33,36,40,43,47,50,55$ and $57(n=5)$. 
Reagents. In situ cell apoptosis detection kit and antibodies against PCNA, cyclin D1, caspase 3 and Bax were purchased from Fuzhou new biotechnology company (Fuzhou Maxim, Fuzhou, China). All other reagents were from Sigma-Aldrich (St. Louis, MO, USA) unless otherwise stated.

Histological analysis. Testes were obtained from mice sacrificed by cervical dislocation and fixed in $10 \%$ formaldehyde, dehydrated in ascending concentrations of ethanol (70-100\%), embedded in paraffin and cut into $3-\mu \mathrm{m}$ sections using a microtome (RM2125RT, Leica Microsystems Trading Ltd., Shanghai, China), mounted and stained with hematoxylin and eosin (H\&E).

TUNEL analysis. Sections of postnatal days 30 and 33 testes were incubated at $60^{\circ} \mathrm{C}$ in the oven for $40 \mathrm{~min}$. Following dewaxing with two washes of xylene, $1 \mathrm{ml} 100 \%$ ethanol was added to remove residual xylene. The sections were then rehydrated with $95,85,70$ and 50\% ethanol for $3 \mathrm{~min}$ at room temperature and then rinsed with PBS. Sections were incubated with pepsin for 15 min at room temperature. Following two 2-min PBS Tween-20 rinses, sections were incubated in $50 \mu \mathrm{l}$ alkaline phosphatase antibody at $37^{\circ} \mathrm{C}$ for $30 \mathrm{~min}$. Sections were washed with PBS three times and incubated for $20 \mathrm{~min}$ at room temperature with 5-bromo-4-chloro-3-indolyl phosphate/nitro blue tetrazolium solution. Following rinsing with PBS three times, sections were labeled with nuclear fast red for $15 \mathrm{~min}$. These sections were visualized under an Olympus inverted microscope (Olympus Corporation, Tokyo, Japan) and representative images were captured. In situ detection of apoptosis-positive cells appeared purple or dark purple and negative cells appeared pink.

Immunohistochemistry. Sections of postnatal day 30 and 33 testes were incubated at $60^{\circ} \mathrm{C}$ in the oven for $40 \mathrm{~min}$. Following dewaxing with two washes of xylene, $1 \mathrm{ml} 100 \%$ ethanol was added to remove residual xylene. The sections were rehydrated with $95,85,70$ and $50 \%$ ethanol for $3 \mathrm{~min}$ at room temperature and rinsed with PBS. Tissue sections were soaked in distilled water to prepare for use. Following antigen retrieval using citrate buffer at high temperature and high pressure, sections were incubated with $50 \mu \mathrm{l} 3 \% \mathrm{H}_{2} \mathrm{O}_{2}$ solution at room temperature for $10 \mathrm{~min}$ to block endogenous peroxidase activity. Sections were then rinsed with PBS three times and non-specific binding was blocked with normal goat serum. Incubation with the primary antibodies was performed at room temperature for $60 \mathrm{~min}$. Sections were washed with PBS three times, incubated with MaxVision ${ }^{\mathrm{TM}}$ rapid immunochemistry reagent (Fuzhou Maxim) for $15 \mathrm{~min}$ at room temperature, washed again and incubated in $0.03 \%$ (w/v) 3,3'-diaminobenzidine with $0.003 \%$ (v/v) hydrogen peroxide until a brown reaction product was observed.

Quantification of multinucleated cell variety in mouse age groups. Five slides were counted from each group under a microscope. To quantify the percentage of seminiferous tubules containing multinucleated cells, six fields of vision were randomly selected under magnification, x200. Following this, 20 seminiferous tubules containing multinucleated cells were selected and the number of multinucleated cells was counted under magnification, $x 400$. Results were presented as mean \pm SEM.

\section{Results}

Histological analysis. Multinucleated cells were not present in the seminiferous tubules at postnatal days 1, 4, 8, 11, 15, 20 and 43 in mouse testicular tissue. In seminiferous sections of tubules in mouse testicular tissues at day 23, 27, 30, 33, 36, 40, 47, 50 and 54, multinucleated cells were observed by a collection of multiple nuclei close to the lumen. The numbers ranged between several and one dozen and the nuclei were as small as the secondary spermatocytes and sperm nucleus and arranged in piles or as a flower ring (Fig. 1).

Immunohistochemistry. PCNA protein immunopositive signal was detected in all seminiferous tubules in postnatal day 30 and 33 mouse testis. PCNA-positive cells were found on the first and second layers of the seminiferous tubules wall. Combined with the results of H\&E staining and judged by cell morphology, the positive cells were reported to be spermatogonia and primary spermatocytes. No positive signals were identified in multinucleated cells (Fig. 2A).

Cyclin D1 protein immunopositive signal was detected in a small number of seminiferous tubules of day 30 and 33 mouse testis. Cyclin D1-positive cells in the seminiferous tubules were located in the first layer of the wall and the positive cells were part of spermatogonial cells. No positive signals were found in multinucleated cells (Fig. 2B).

A marked immunopositive signal of Bax protein was detected in all seminiferous tubules of day 30 and 33 mouse testis. Bax-positive cells located between layers 1 and 6 had strong immunoreactive signals and were also found in multinucleated cells. The positive cells may be spermatogonia, primary spermatocytes, secondary spermatocytes, round spermatids and multinucleated cells (Fig. 2C).

Caspase 3 protein immunoreactive signal was detected in a small number of seminiferous tubules in day 30 and 33 mouse testis. Caspase 3-positive cells were scattered in each layer of the seminiferous tubules and a marked immunoreactive signal was detected in multinucleated cells. Cell morphology revealed that the positive cells were multinucleated cells, spermatogonia, primary spermatocytes, secondary spermatocytes and round spermatids (Fig. 2D).

In situ cell apoptosis detection. In day 33 testicular tissue, spermatogonia, primary spermatocytes, secondary spermatocytes and sperm cells exhibited a strong apoptotic signal and were detected in seminiferous tubules. Multinucleated cells did not reveal a positive apoptotic signal (Fig. 3).

Quantification of multinucleated cells. The ratio of the seminiferous tubules containing multinucleated cells in mouse testis indicated that the rates from days 1 to 20 were 0 (Table I and Fig. 4). The appearance of multinucleated cells in mouse testis seminiferous tubules began at day 23 and the rate was maintained at an elevated level between days 23 and 33. It began to decline at day 36 and remained at a lower level in the cell phases that followed (Table I and Fig. 4). Quantification of multinucleated cells in the seminiferous tubules indicated that multinucleated cell numbers peaked at day 33 and were maintained at a relatively stable state in the remaining phases (Table I and Fig. 5). 
Table I. Quantification of multinucleated cells of mouse testis from various age groups.

\begin{tabular}{|c|c|c|}
\hline $\begin{array}{l}\text { Age of the mouse } \\
\text { (postnatal days) }\end{array}$ & $\begin{array}{l}\text { Ratio of seminiferous tubules } \\
\text { containing multinucleated cells } \\
\text { (mean } \pm \text { SEM })\end{array}$ & $\begin{array}{l}\text { Ratio of number of multinucleated } \\
\text { cells in seminiferous tubules } \\
(\text { mean } \pm \text { SEM })\end{array}$ \\
\hline 1 & 0 & 0 \\
\hline 4 & 0 & 0 \\
\hline 8 & 0 & 0 \\
\hline 11 & 0 & 0 \\
\hline 15 & 0 & 0 \\
\hline 20 & 0 & 0 \\
\hline 23 & $0.38 \pm 0.055$ & $2.20 \pm 0.515$ \\
\hline 27 & $0.25 \pm 0.030$ & $1.30 \pm 0.335$ \\
\hline 30 & $0.28 \pm 0.005$ & $2.10 \pm 0.280$ \\
\hline 33 & $0.32 \pm 0.010$ & $4.90 \pm 0.595$ \\
\hline 36 & $0.10 \pm 0.015$ & $2.80 \pm 0.770$ \\
\hline 40 & $0.12 \pm 0.010$ & $1.80 \pm 0.315$ \\
\hline 43 & 0 & 0 \\
\hline 47 & $0.02 \pm 0.020$ & $1.67 \pm 0.285$ \\
\hline 50 & $0.02 \pm 0.015$ & $2.00 \pm 0.705$ \\
\hline 54 & $0.08 \pm 0.035$ & $1.80 \pm 0.390$ \\
\hline 57 & 0 & 0 \\
\hline
\end{tabular}
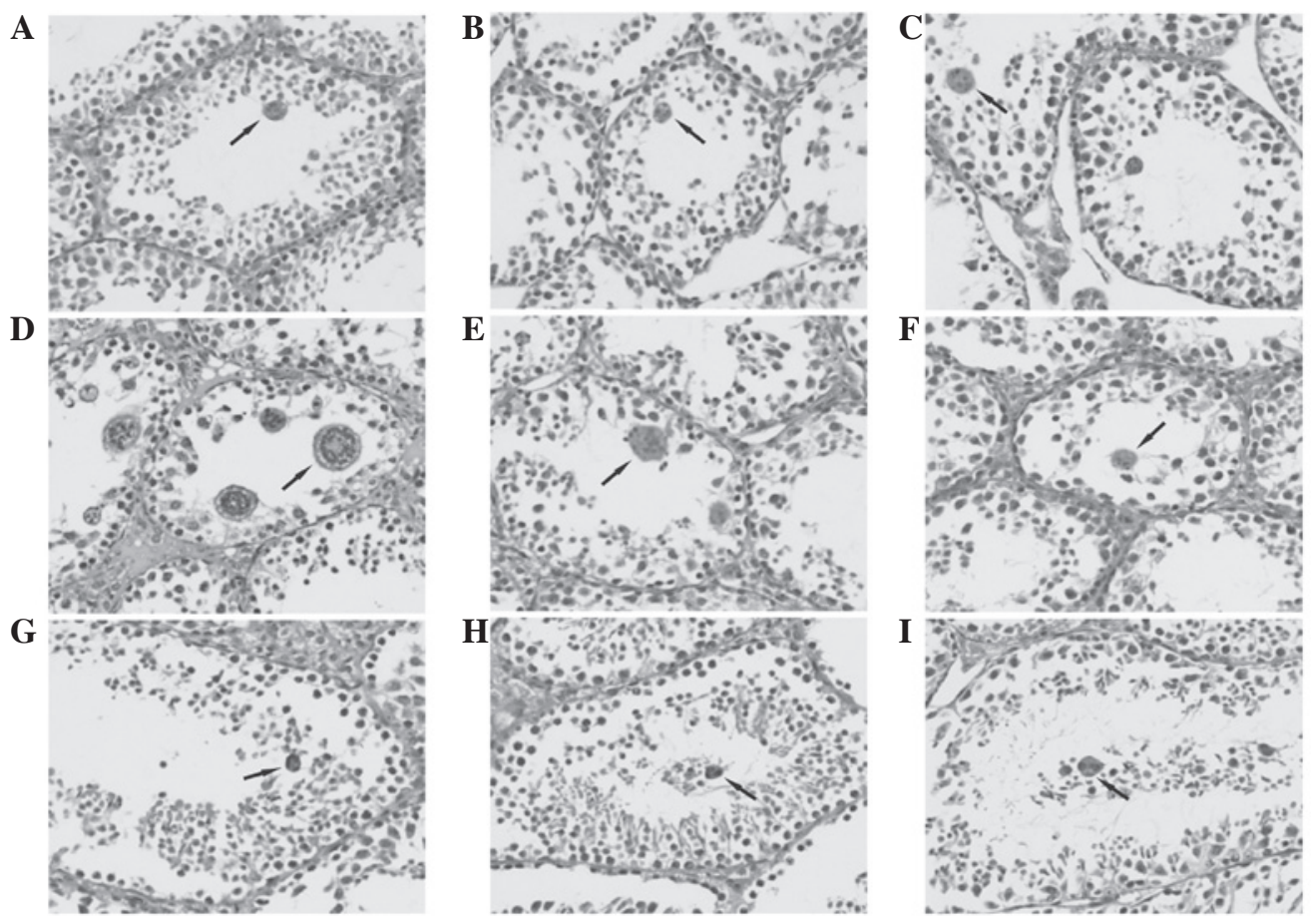

Figure 1. H\&E staining results of multinucleated cells in seminiferous tubules of mouse testis at various developmental stages (magnification, $\mathrm{x} 200$ ). Days (A) 23, (B) 27, (C) 30, (D) 33, (E) 36, (F) 40, (G) 47, (H) 50 and (I) 54. Arrows show multinucleated cells. H\&E, hematoxylin and eosin.

\section{Discussion}

The current study evaluated multinucleated cell appearance and potential roles during normal testicular development. Multinucleated cells have been described as being present primarily in pathological processes (10). To the best of our knowledge, the present study has, for the first time, shown that during normal development of the mouse testis, multinucleated cells appeared in sections of the seminiferous tubules at postnatal days $23,27,30,33,36,40,47,50$ and 54 , suggesting 


\section{A}

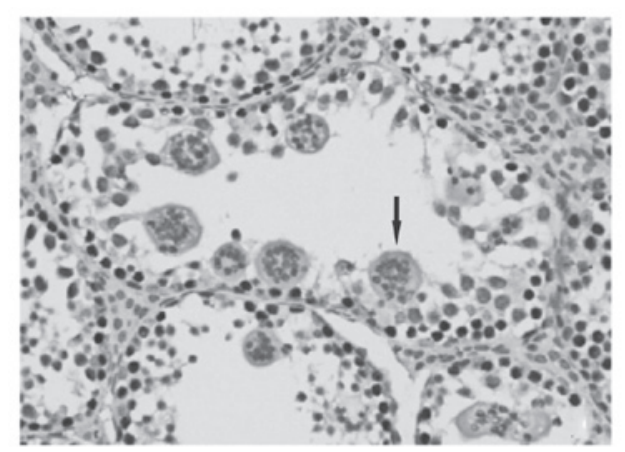

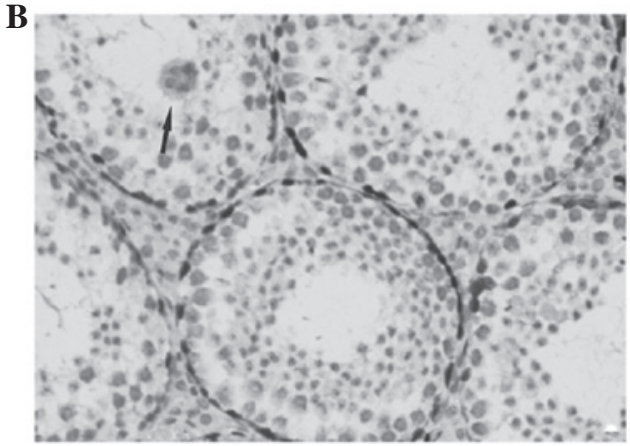

$\mathrm{C}$

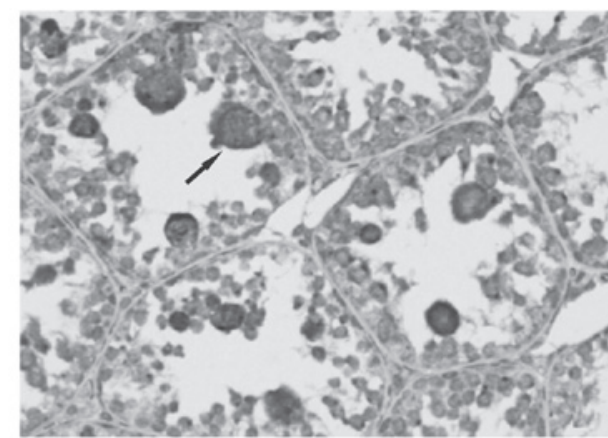

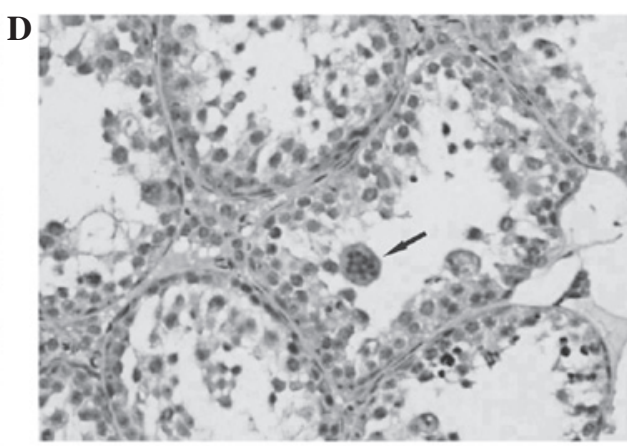

Figure 2. Immunostaining results of multinucleated cells in seminiferous tubules of mouse testis (magnification, x200). (A) PCNA, (B) cyclin D1, (C) Bax and (D) caspase 3. Arrows indicate multinucleated cells. PCNA, proliferating cell nuclear antigen.

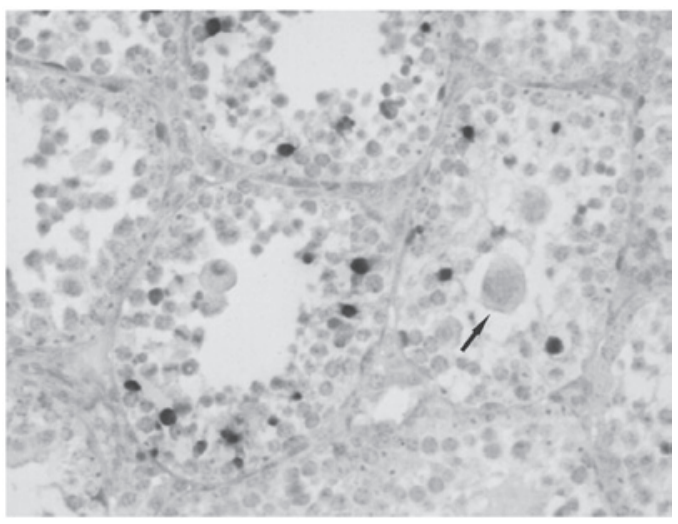

Figure 3. In situ apotosis detection of multinucleated cell apoptosis in day 33 mouse testis seminiferous tubules (magnification, x200). Arrows show multinucleated cells.

that multinucleated cells exist in various stages of the normal developmental process in mouse testis. Given this phenomenon, it is hypothesized that multinucleated cells appear in pathological processes and in normal testicular development.

Multinucleated cells were identified between postnatal days 23 and 33 and peaked at day 33. The first development and maturation time of the Kunming mouse spermatogenic cell occurs $\sim 5$-weeks following birth (11), suggesting that the peak of the multinucleated cells occurred prior to the first maturity period of the sperm cell. Multinucleated cells were first observed at postnatal day 23 in the center of the seminiferous tube cavity with similar nucleus size as secondary spermatocytes and spermatids. Our previous study revealed that secondary spermatocytes and a small number of round spermatids may first be observed on postnatal day 23 in the seminiferous tubules of the mouse (12). Therefore, given the time, location and nuclear morphology, these multinucleated cells may be derived from the secondary spermatocytes and spermatids.

Following this, the potential role of multinucleated cells during mouse testicular development was investigated. Spermatogenesis is the process by which SSCs self-renew and differentiate into sperm. The role of multinucleated cells in SSC proliferation and differentiation was analyzed. During the cell cycle, cyclin D1 combines with cyclin-dependent kinase (CDK) 4 or CDK6 to phosphorylate downstream retinoblastoma protein (RB) and its associated proteins, P107 and P130. Phosphorylated RB may relieve inhibition of the E2F transcription factor and initiate DNA replication, driving the cell cycle from $\mathrm{G} 1$ to $\mathrm{S}$ phase $(13,14)$. In the present study, cyclin D1 was expressed in the spermatogonia. Expression of cyclin D1 during active cell cycle is consistent with its role in promoting the transition of the cell cycle from $\mathrm{G} 1$ to $\mathrm{S}$ phase.

PCNA is a cofactor in eukaryotic DNA polymerase $\delta$, which plays a role in the $\mathrm{S}$ phase of DNA synthesis and repair and indicates cell proliferation (15). In the current study, PCNA was expressed in spermatogonia and primary spermatocytes, consistent with a previous study by Zhang et al which demonstrated that PCNA plays a role in spermatogonia and primary spermatocytes of the DNA replication process (16).

Immunostaining revealed no positive immune signals in multinucleated cells, indicating that cyclin D1 and PCNA was not expressed in these cells. The observations indicated no physiological and biochemical activities of the G1 or S-phase appearing within the cells with no DNA replication occurring. Therefore, it was hypothesized that multinucleated cells are not involved in the cell cycle and the cell proliferation process.

In typical spermatogenesis, spermatids undergo proliferation and degradation, the latter is achieved mainly through 


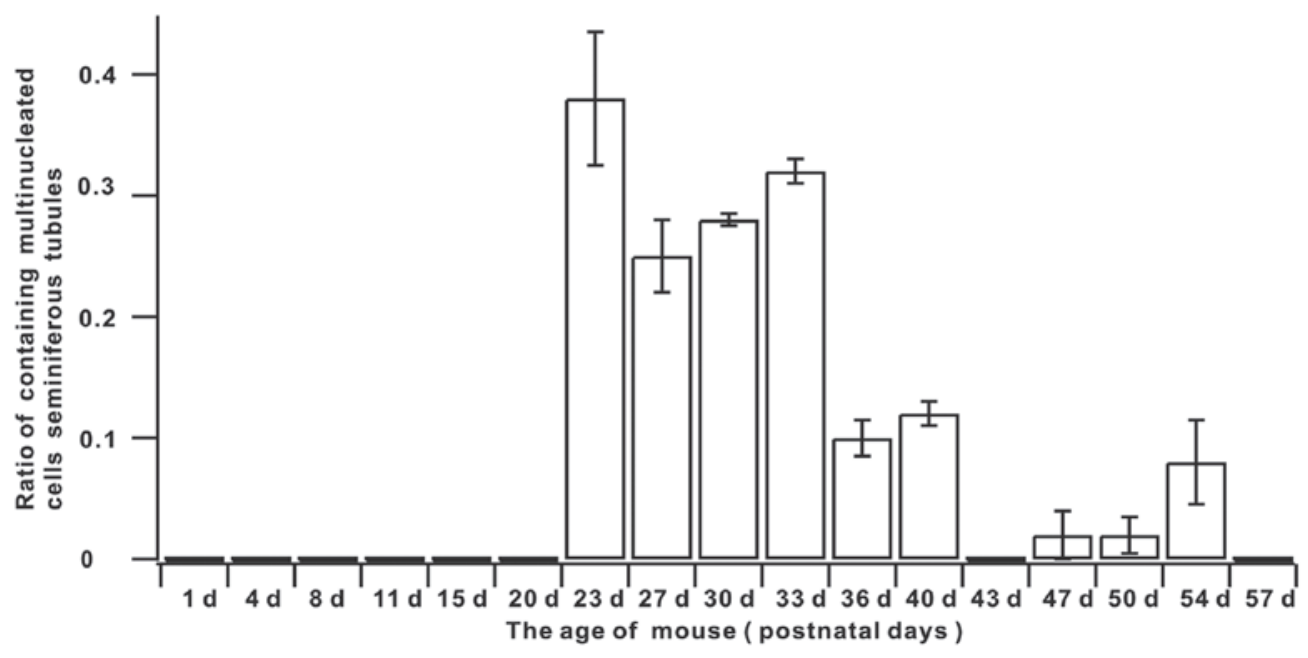

Figure 4. Ratio of seminiferous tubules containing multinucleated cells in normal mouse testis development.

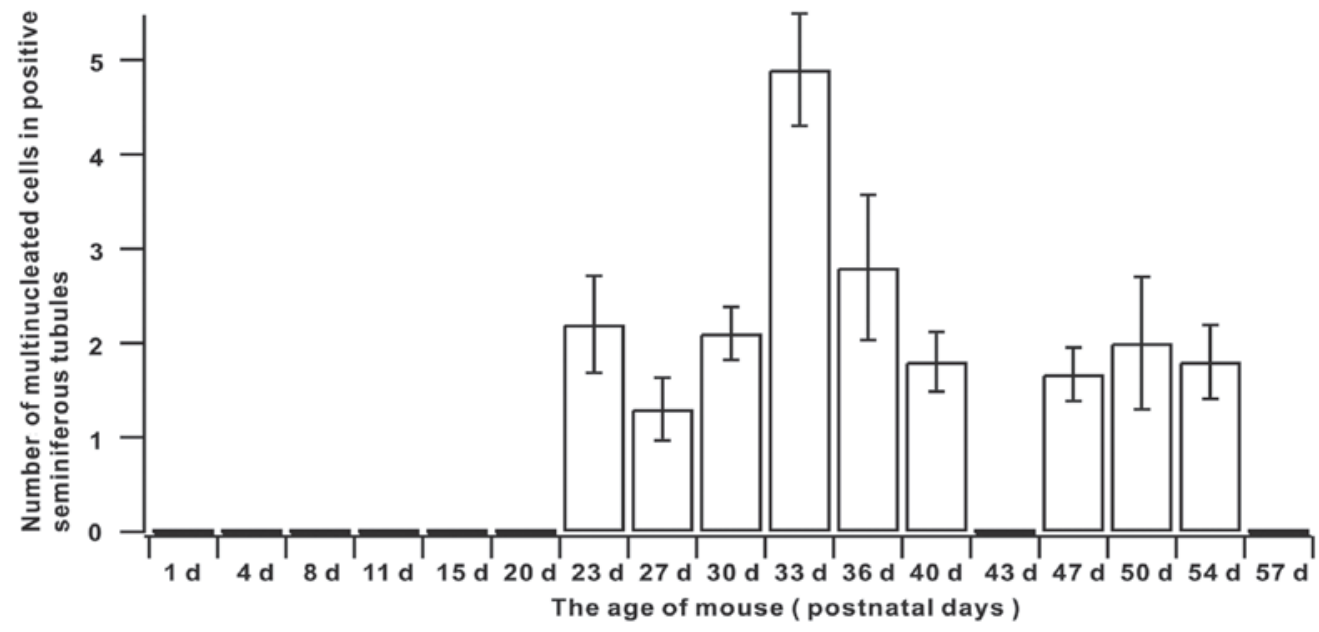

Figure 5. Number of multinucleated cells in seminiferous tubules during mouse testis development.

apoptosis. Apoptosis of spermatogenic cells ensures the quantity and quality of spermatogenic cell survival (17).

Bax is an apoptosis-inducing factor, mainly located in the cytoplasm when apoptosis occurs. Bax inserts into the mitochondrial membrane causing cytochrome $\mathrm{c}$ release from the cytoplasm which induces apoptosis in spermatogenic cells $(18,19)$. Immunostaining revealed that the Bax protein immunoreactive signal was detected in specific spermatogonia, primary spermatocytes, secondary spermatocytes and round spermatids. This is consistent with a previous study by Rodriguez et al which revealed that prior to normal mouse testis maturation, a temporary elevated expression stage of the Bax gene was noted (20). Findings of a study by Beumer et al showed that all cell types expressed Bax protein in mouse testes (21).

Caspase 3 is a important apoptosis effector in the middle and later phases of apoptosis $(22,23)$. Immunostaining results of the present study revealed that caspase 3 was expressed in individual spermatogonia, primary spermatocytes, secondary spermatocytes and round spermatids. In situ apoptosis detec- tion indicated that a small number of spermatogenic cells appear to undergo apoptosis. This observation confirms that, compared with Bax, caspase 3 expression is consistent with apoptosis of spermatogenic cells in terms of time and space.

Immunostaining also revealed immunoreactive Bax and caspase 3 signals in the nuclei of multinucleated cells, indicating that Bax and caspase 3 are involved in the formation and apoptosis of multinucleated cells. Thus, multinucleated cells may be associated with apoptotic processes.

However, the observations from in situ apoptosis detection, performed during the same period, failed to produce a positive apoptotic signal in multinucleated cells, indicating that apoptotic events did not arise in multinucleated cells. Further investigation is required to determine whether apoptosis occurs later following the expression of apoptosis-associated genes or whether expression of these genes does not result in apoptosis in these cells.

In summary, this study has to the best of our knowledge demonstrated, for the first time, that multinucleated cells are present during normal testicular development and may be 
associated with SSC apoptosis. Therefore, multinucleated cells may be important in the spermatogenesis process.

\section{Acknowledgements}

This study was supported by grants from the Applied Basic Research Joint Foundation of Yunnan Provincial Science and Technology department and Kunming medical university (Study on mechanism of formation and clearance of multinucleated cells in development in mouse testes, 2013, for Lan Luo), Research Foundation of the Department of Education of Yunnan Province (no.2011Y165), National Natural Science Foundation of China (31260264, 81060034, 81060126 and 31260243 ). The study was also supported by Chunhui Project (Z2011047), State Education Ministry (SEM) and Program for New Century Excellent Talents in University 'NCET' for Dr Yin Wang.

\section{References}

1. Johnston DS, Wright WW, Dicandeloro P, Wilston E, Kopf GS and Jelinsky SA: Stage-specific gene expression is a fundamental characteristic of rat spermatogenic cells and Sertoli cells. Proc Natl Acad Sci USA 105: 8315-8320, 2008.

2. Kokkinaki M, Lee TL, He ZP, Jiang JJ, Golestaneh N, Hofmann MC, Chan WY and Dym M: Age affects gene expression in mouse spermatogonial stem/progenitor cells. Reproduction 139: 1011-1020, 2010.

3. D'Souza UJ: Tamoxifen induced multinucleated cells (symplasts) and distortion of seminiferous tubules in rat testis. Asian J Androl 5: 217-220, 2003.

4. Boekelheide K, Kleymenova E, Liu KJ, Swanson C and Gaido KW: Dose-dependent effects on cell proliferation, seminiferous tubules and male germ cells in the fetal rat testis following exposure to di(n-butyl) phthalate. Microsc Res Tech 72: 629-638, 2009.

5. Kondarewicz A, Kolasa A, Zawiślak B, Baranowska-Bosiacka I, Marchlewicz M, Wenda-Różewicka L and Wiszniewska B: Testis morphology in rats chronically treated with letrozole, an aromatase inhibitor. Folia Histochem Cytobiol 49: 677-684, 2011.

6. Ramzan F and Qureshi IZ: Intraperitoneal kisspeptin-10 administration induces dose-dependent degenerative changes in maturing rat testes. Life Sci 88: 246-256, 2011.

7. Anton E: Arrested apoptosis without nuclear fragmentation produced by efferent duct ligation in round spermatids and multinucleated giant cells of rat testis. Reproduction 125: 879-887, 2003.

8. Schofield JB and Evans DJ: Multinucleate giant stromal cells in testicular atrophy following oestrogen therapy. Histopathology 16 : 200-201, 1990
9. Coyne JD and Dervan PA: Multinucleated stromal giant cells of testis. Histopathology 31: 381-383, 1997.

10. Pitt MA, Roberts IS, Agbamu DA and Eyden BP: The nature of atypical multinucleated stromal cells: a study of 37 cases from different sites. Histopathology 23: 137-145, 1993.

11. Yu J, Wan HJ, Cai ZM, Fang JZ, Zhang FT, Ye J and Yin MJ: The histological observation of the stepwise development of neonatal mouse testis in immunodeficient mice. Jie Pou Xue Bao 38: 213-217, 2007 (In Chinese).

12. Luo L, Zhang Y and Yang F: The histological observation in the testis of mice at different development stage. Laboratory Animal Science 27: 10-13, 2010 (In Chinese).

13. Beumer TL, Roepers-Gajadien HL, Gademan IS, Kal HB and de Rooij DG: Involvement of the D-type cyclins in germ cell proliferation and differentiation in the mouse. Biol Reprod 63: 1893-1898, 2000.

14. Baker GL, Landis MW and Hinds PW: Multiple functions of D-type cyclins can antagonize $\mathrm{pRb}$-mediated suppression of proliferation. Cell Cycle 4: 330-338, 2005.

15. Zusman I, Reifen R, Livni O, Smirnoff P, Gurevich P, Sandler B, Nyska A, Gal R, Tendler Y and Madar Z: Role of apoptosis, proliferating cell nuclear antigen protein in chemically induced colon cancer in rats fed corncob fiber treated with the fungus Pleurotus ostreatus. Anticancer Res 17: 2105-2113, 1997.

16. Zhang J, Gao FL, Zhi HY and Duan XG: Age-specific changes of proliferation and apoptosis in the seminiferous epithelium of laboratory mice. Dong Wu Xue Bao 47: 209-214, 2001 (In Chinese).

17. de Rooij DG: Stem cells in the testis. Int J Exp Pathol 79: 67-80, 1998.

18. Eskes R, Desagher S, Antonsson B and Martinou BA: Bid induces the oligomerization and insertion of Bax into outer mitochondrial membrane. Mol Cell Biol 20: 929-935, 2000.

19. Antonsson B, Montessuit S, Sanchez B and Martinou JC: Bax is present as a high molecular weight oligomer/complex in the mitochondrial membrane of apoptotic cells. J Biol Chem 276: 11615-11623, 2001.

20. Rodriguez I, Ody C, Araki K, Garcia I and Vassalli P: An early and massive wave of germinal cell apoptosis is required for the development of functional spermatogenesis. EMBO J 16: 2262-2270, 1997.

21. Beumer TL, Roepers-Gajadien HL, Gademan IS, Lock TM, Kal HB and de Rooij DG: Apoptosis regulation in the testis: involvement of Bcl-2 family members. Mol Reprod Dev 56: 353-359, 2000

22. Almeida C, Cunha M, Ferraz L, Silva J, Barros A and Sousa M: Caspase-3 detection in human testicular spermatozoa from azoospermic and non-azoospermic patients. Inter J Androl 34: e407-e414, 2011.

23. Philchenkov AA: Caspases as regulators of apoptosis and other cell functions. Biochemistry (Mosc) 68: 365-376, 2003. 